\title{
Ultrasound and CT Evaluation in Renal Failure
}

\section{Dr. Moscow Mani S A MBBS MD*}

Radiologist, Bangalore Urban, Karnataka, India

*Corresponding Author: Dr. Moscow Mani S A MBBS MD, Radiologist, Bangalore

Urban, Karnataka, India.
Received: March 25, 2021

Published: June 23, 2021

(C) All rights are reserved by Dr. Moscow Mani S A MBBS MD.

\begin{abstract}
Background: Acute renal failure remains a vexing and significant clinical problem. Mortality from ARF is still elevated and has changed little over time despite technical advances in renal replacement therapy and supportive care, possibly because of increasing age and increasing co-morbidity of patients. Hospitalizations for ARF have dramatically increased in the past two decades. ARF may also be a precursor to ESRD as approximately $13 \%$ of patients with ARF proceed to ESRD over a 3-yr period.

Methods: In this Prospective study, we analyzed 65 case histories of patients hospitalized with elevated serum creatinine. Ultrasound echocity and CT attenuation values were noted.

Results: In our series of 65 cases, 45 (69.2\%) were males and 20 (30.8\%) were females .Our study shows greater incidence of ARF among males than females. The maximum incidence of ARF in this series was found to be between 50 - 59 years. 15 patients (23.1\%) presented with ARF in the $5^{\text {th }}$ decade.

- In our study, abdominal ultrasound was done in all the patients, 47 (72.3\%) patients had normal size kidneys, 15 (23.07\%) had small size kidneys, and 3 (4.61\%) had large sized kidneys. In our study there was no significant correlation between renal length and serum creatinine levels.

- There was a significant correlation between renal length and age group of the patient, wherein there was a slight decrease in the renal lengths in the forty and above age group patients.

- In this series, when the cortical echogenicity was correlated with laboratory data, an overall significant correlation was found between cortical echogenicity and the serum creatinine levels.

- In our study, sixty four patients had normal parenchymal attenuation value in both kidneys, except for one who had a obstructive ureterolithiasis on the right side. Renal attenuation on the side with lithiasis was lower compared to the opposite kidney. However, in our study there was no significant correlation between renal parenchymal attenuation and serum creatinine levels.

Conclusion: Our prospective study with 65 adult patients revealed that acute renal failure had a positive association with male gender and there was a gradual decrease in renal length with advancing age. The renal cortical echogenicity on ultrasound had a positive relation with serum creatinine levels and reflected the severity of the disease process. Non-enhanced computer tomography is a more sensitive modality in detecting other pathologies in the kidney, in particular renal and ureteric calculus. It also demonstrated a decrease in renal parenchymal attenuation in the obstructed kidney. The limited responses of the kidney to various pathological insults may well remain a limitation to specific sonographic diagnosis, even when accurate quantitative measurements by ultrasound become available. Sonographic characterization of tissue is at present an area of active research. Measurements of the degree of abnormality and the definition of unique tissue signatures are the goals.
\end{abstract}

Keywords: Computer Tomography; Kidney; Ultrasound 


\section{Abbreviations}

ARF: Acute Renal Failure; CRF: Chronic Renal Failure; ATN: Acute Tubular Necrosis; CT: Computer Tomography; ESRD: End Stage Renal Failure; HU: Hounsefield Unit; LK: Left Kidney; mg/dl: Milligram per Deciliter; RK: Right Kidney; ROI: Region of Interest; VAR: Variance

\section{Introduction}

Acute renal failure remains a vexing and significant clinical problem. Mortality from ARF is still elevated and has changed little over time despite technical advances in renal replacement therapy and supportive care, possibly because of increasing age and increasing co-morbidity of patients. Hospitalizations for ARF have dramatically increased in the past two decades. ARF may also be a precursor to ESRD as approximately $13 \%$ of patients with ARF proceed to ESRD over a 3-yr period.

Sonography is a critical component in the evaluation of both acute and chronic renal failure; however, most nephrologists have a limited knowledge of this procedure. The acoustic properties, limited spectrum of pathological changes, and ease of visualization of the kidneys, coupled with the safety, simplicity, and low cost of sonography, make it the modality of choice for renal imaging.

Although many sonographic findings are nonspecific, their diagnostic use is greatly enhanced by a familiarity with the clinical presentation and a thorough understanding of renal pathophysiological characteristics. Therefore, nephrologists should be knowledgeable about renal sonography and participate in its interpretation.

Ultrasound is usually the first imaging procedure used to evaluate the kidneys in a patient presenting with renal failure. It can allow the physician to make a specific diagnosis of bilateral hydronephrosis as the cause of the renal failure and irreversible end-stage renal disease when bilaterally small echogenic kidneys are seen.

Computed tomography has become an essential tool for diagnosis in virtually all areas of the body. Computed tomography scanning in ARF and CRF usually follows ultrasound evaluation that demonstrated bilateral hydronephrosis. Even in areas where ultrasound is employed, CT offers a complementary and sometimes superior means of imaging.

Computed tomography is now the first examination to be performed in patients with renal colic, renal stone disease, renal trauma, renal infection and abscess, renal mass, hematuria, and finally, urothelial abnormalities. Noncontrast CT easily demonstrates the dilated pelvocalyceal systems in the kidney. The parenchymal thickness relative to the dilated collecting systems can be visualized.

State-of-the-art magnetic resonance imaging (MRI) presently allows for enhanced resolution of regional renal blood flow and functional evaluations in patients. Furthermore, new probes and techniques for MRI that allow for identification and quantitation of inflammation, applicable to human ARF, are being developed and tested in animal models.

\section{Materials and Methods}

This prospective study was performed in the department of radio-diagnosis and imaging, K.G. hospital and postgraduate medical institute, Coimbatore in the period between January 2016 - December 2016. The ethical committee of the hospital approved the study.

\section{Patient selection}

Adult patients who were diagnosed to be in acute renal failure were referred from the department of nephrology for imaging evaluation of the kidneys.

\section{Inclusion criteria}

- Patients aged 18 years or older and

- Documented acute renal failure, as evidenced by a serum creatinine level of $1.5 \mathrm{mg} / \mathrm{dl}$ or greater.

\section{Exclusion criteria}

- Patients aged younger than 18 years,

- Prior renal transplant,

- Diagnosed end stage renal failure,

- Current pregnancy,

- Serum creatinine level of less than $1.5 \mathrm{mg} / \mathrm{dl}$,

- Evidence of hepatic or splenic disease.

A total of 65 adult patients were included in our study. Of the patients, 45 were males and 20 were females, with ages ranging from 19 to 89 years. These patients were evaluated by ultrasound and by computed tomography.

\section{Equipment's}

All patients were evaluated using GE Voluson-730pro ultrasound machine and by Wipro GE CT/e spiral CT scanner. 
Methods

Imaging protocol to asses the renal length and echogenicity by ultrasound

The ultrasound studies were performed with a GE Voluson 730 pro unit by using a 2-5 MHz convex transducer. The examination was started with the patient in the supine position, if necessary, the patients were scanned from a lateral or posterolateral view-which ever approach enabled optimal visualization of the kidney.

To study the right kidney, the liver was used as a window in the supine patient. Scans of the left kidney were obtained using the spleen as a window with the patient in the decubitus position.

The maximum bipolar lengths of the kidneys were measured in the longitudinal plane and were visually estimated to represent the largest longitudinal section. A mean absolute kidney length was calculated using the sum of the right and left kidney length divided by two.The average renal length of $9-13 \mathrm{cms}$ was considered as normal, $<9 \mathrm{cms}$ was considered as small and $>13 \mathrm{cms}$ was considered as enlarged.

The echogenicity of the right and left renal cortex was assessed by comparing it with that of the liver or spleen respectively. Normally, the echogenicity of the renal cortex is less than that of the adjacent organs.

The renal cortical echogenicity was graded by comparing the amplitude of echoes in the renal cortex with that of the adjacent liver, spleen, and renal sinus:

- Grade 0: Echogenicity less than liver or spleen.

- Grade 1: Echogenicity equal to liver or spleen.

- Grade 2: Echogenicity greater than liver or spleen but less than that of renal sinus.

- Grade 3: Echogenicity equal to that of renal sinus.

In addition to the size and echogenicity of the kidney, other potentially useful information provided by ultrasound was recorded.

Imaging protocol to assess the renal parenchymal attenuation by CT

All scans were obtained on a single detector scanner (Wipro GE CT/e high speed spiral scanner), using a helical technique (collimation, $5 \mathrm{~mm}$; pitch, 1.0) from the level of the T12 vertebral body to the pubic symphysis in one breath- hold. Scans were acquired using 175 - $350 \mathrm{mAs}$ at $120 \mathrm{kVp}$. Neither oral nor IV contrast material was administered.

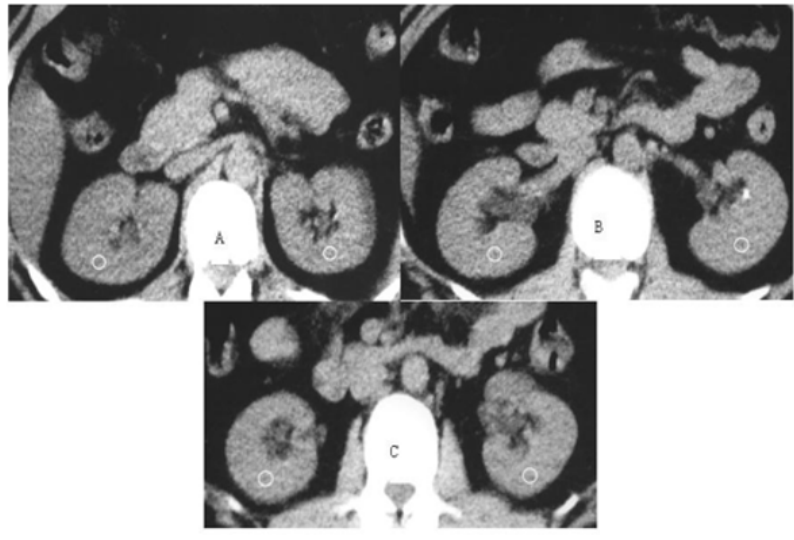

Figure a: Unenhanced CT scan shows parenchymal (corticomedullary) placement of elliptical region of interest (ROI) to measure attenuation values in A) upper B) middle and C) lower.

Attenuation values were systematically measured with an elliptic region of interest (ROI) in the upper, middle, and lower portions of renal parenchyma (corticomedullary) (soft-tissue window: center, $20 \mathrm{H}$; width, $300 \mathrm{H}$ ). In the upper pole, the ROI was placed in the first section that depicted the collecting system. In the middle portion, the measurement was made at the level of the hilum and, in the lower pole; the ROI was placed in the last section in which the collecting system could be identified. All measurements were made with a similar-sized ROI $\left(=40 \mathrm{~mm}^{2}\right)$ in the posterior region of the kidney parenchyma. A mean attenuation value (in Hounsfield units) was calculated for each kidney from the measurement.

\section{Statistical analysis}

After all the data were obtained, statistical analysis of the independent results was performed using SPSS version 13.0 software. The variables studied were:

- Sex and age prevalence of ARF

- Renal length: The renal length was correlated with laboratory finding using t-test and Pearsons correlation.

- Cortical echogenicity: The cortical echogenicity was correlated with the laboratory finding using t-test and Pearsons correlation.

- Parenchymal attenuation: The parenchymal attenuation was correlated with the laboratory finding using t-test and Pearsons correlation. 


\section{Results}

Our study consisted of 65 patients who were diagnosed to have acute renal failure by laboratory parameters. Ultrasound and CT was performed on them to evaluate the kidneys.

Sex distribution of ARF

\begin{tabular}{|l|c|c|}
\hline \multicolumn{1}{|c|}{ Sex } & Frequency & Percent \\
\hline Male & 45 & 69.2 \\
Female & 20 & 30.8 \\
\hline Total & 65 & 100.0 \\
\hline
\end{tabular}

Table 1

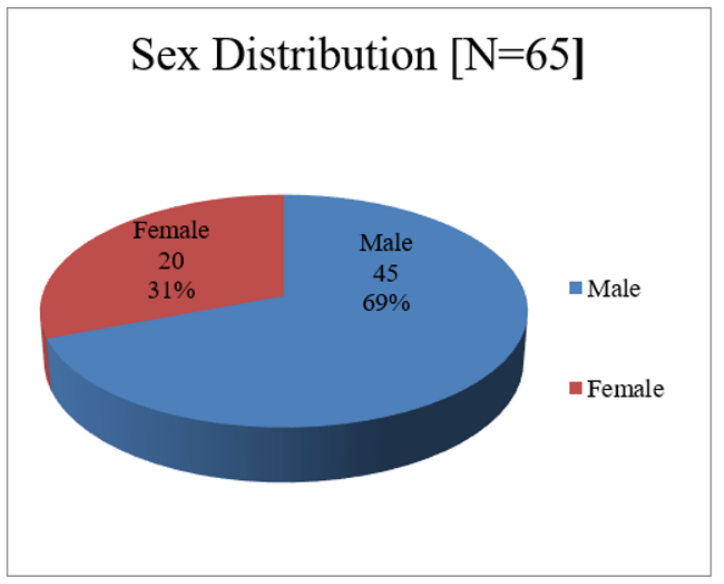

Figure b

Age group distribution of ARF

\begin{tabular}{|l|c|c|}
\hline Age group (years) & Total & Percentage \\
\hline $19-29$ & 7 & 10.8 \\
\hline $30-39$ & 6 & 9.2 \\
\hline $40-49$ & 10 & 15.4 \\
\hline $50-59$ & 15 & 23.1 \\
\hline $60-69$ & 12 & 18.5 \\
\hline $70-79$ & 11 & 16.9 \\
\hline $80-89$ & 4 & 6.2 \\
\hline Total & 65 & \\
\hline
\end{tabular}

Table 2

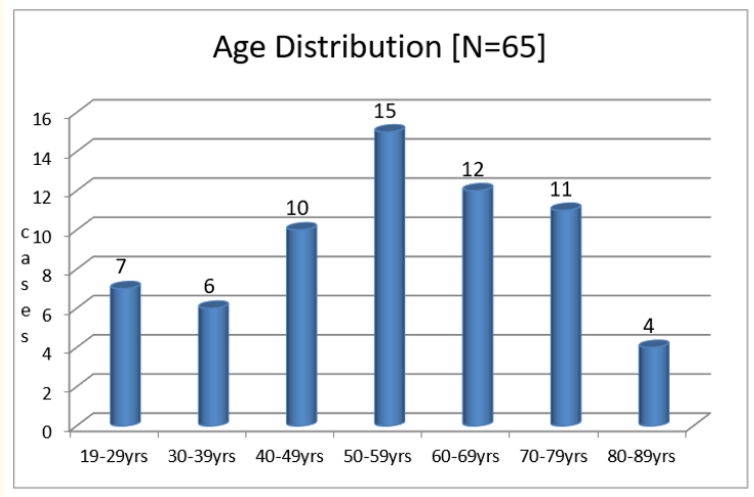

Figure c

\begin{tabular}{|l|c|c|}
\hline \multicolumn{1}{|c|}{ Age group } & Frequency & Percent \\
\hline Less than 40 years & 14 & 21.5 \\
More than 40 years & 51 & 78.5 \\
\hline Total & 65 & 100 \\
\hline
\end{tabular}

Table 3

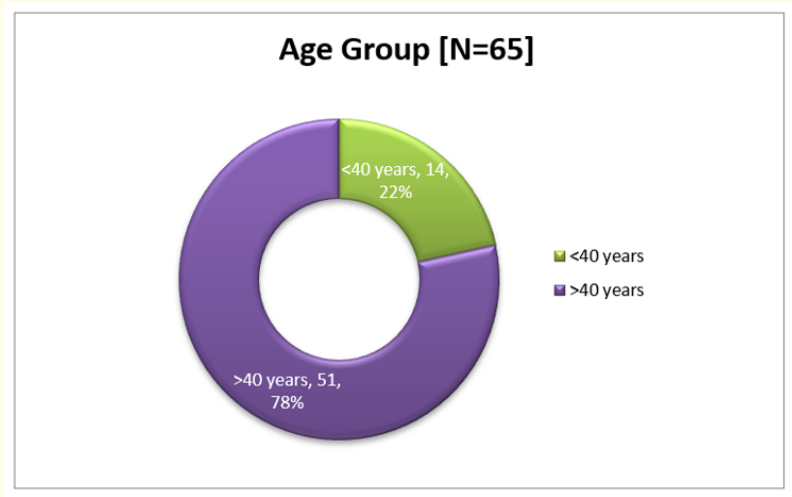

Figure d

In this series majority of the patients with ARF belonged to the forty and above age group. 
Age and sex distribution of ARF

\begin{tabular}{|l|c|c|c|}
\hline \multirow{2}{*}{$\begin{array}{c}\text { Age group } \\
\text { (years) }\end{array}$} & \multicolumn{2}{|c|}{ Gender } & \multirow{2}{*}{ Total } \\
\cline { 2 - 3 } & Male & Female & \\
\hline $19-29$ & 6 & 1 & 7 \\
\hline $30-39$ & 5 & 1 & 6 \\
\hline $40-49$ & 5 & 5 & 10 \\
\hline $50-59$ & 8 & 7 & 15 \\
\hline $60-69$ & 7 & 5 & 12 \\
\hline $70-79$ & 11 & 0 & 11 \\
\hline $80-89$ & 3 & 1 & 4 \\
\hline Total & 45 & 20 & 65 \\
\hline
\end{tabular}

Table 4

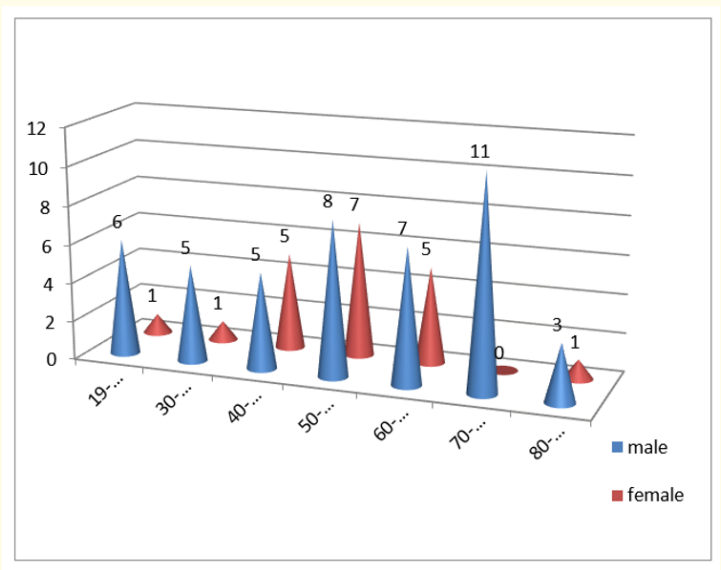

Figure e

Ultrasound and CT findings

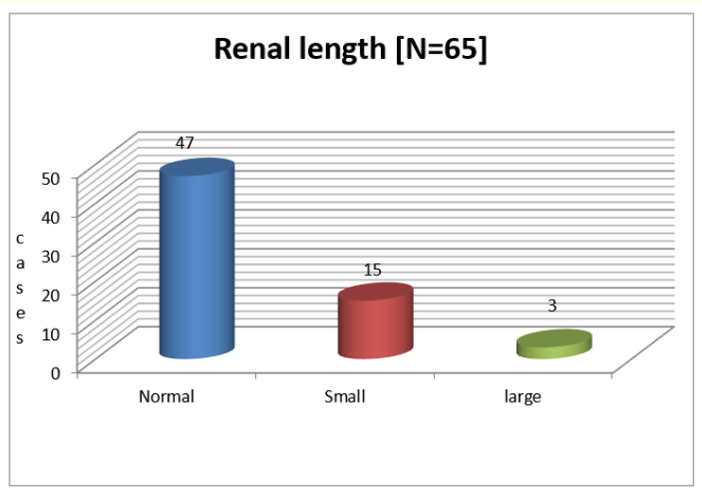

\begin{tabular}{|l|c|c|}
\hline \multicolumn{3}{|c|}{ Renal size (length) on ultrasound } \\
\hline \multicolumn{1}{|c|}{ Parameters } & Case distribution & Percentage \\
\hline Normal & 47 & $72.3 \%$ \\
\hline Small & 15 & $23.07 \%$ \\
\hline Large & 3 & $4.61 \%$ \\
\hline
\end{tabular}

Table 5: Distribution of cases according to renal length.

\begin{tabular}{|l|c|c|}
\hline \multicolumn{3}{|c|}{ Renal cortical echogenicity on ultrasound } \\
\hline Parameters & Case distribution & Percentage \\
\hline Grade 0 & 40 & $61.53 \%$ \\
\hline Grade 1 & 11 & $16.92 \%$ \\
\hline Grade 2 & 9 & $13.84 \%$ \\
\hline Grade 3 & 5 & $7.7 \%$ \\
\hline
\end{tabular}

Table 6: Distribution of cases according to grades of cortical echogenicity.

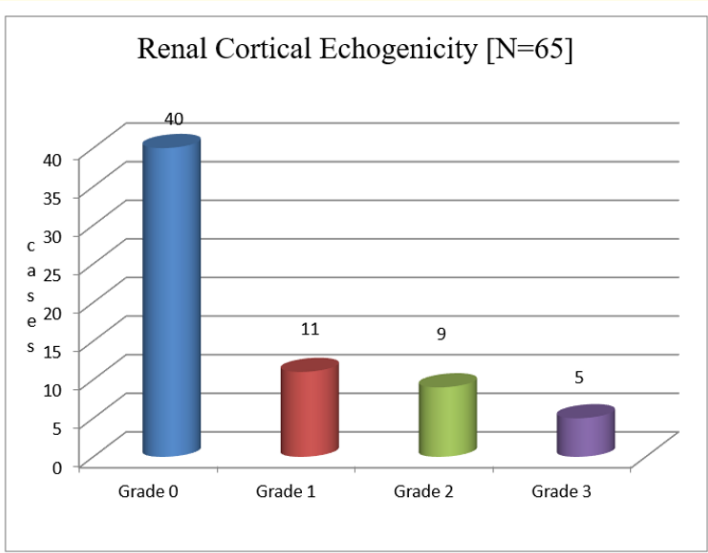

Figure g

\begin{tabular}{|l|c|c|}
\hline \multicolumn{3}{|c|}{ Renal parenchymal attenuation on CT } \\
\hline \multicolumn{1}{|c|}{ Parameters } & Right kidney & Left kidney \\
\hline Normal & 64 & 65 \\
\hline Decreased & 1 & 0 \\
\hline Increased & 0 & 0 \\
\hline
\end{tabular}

Table 7: Distribution of cases according to renal parenchymal attenuation.

Figure $\mathbf{f}$ 
Correlations

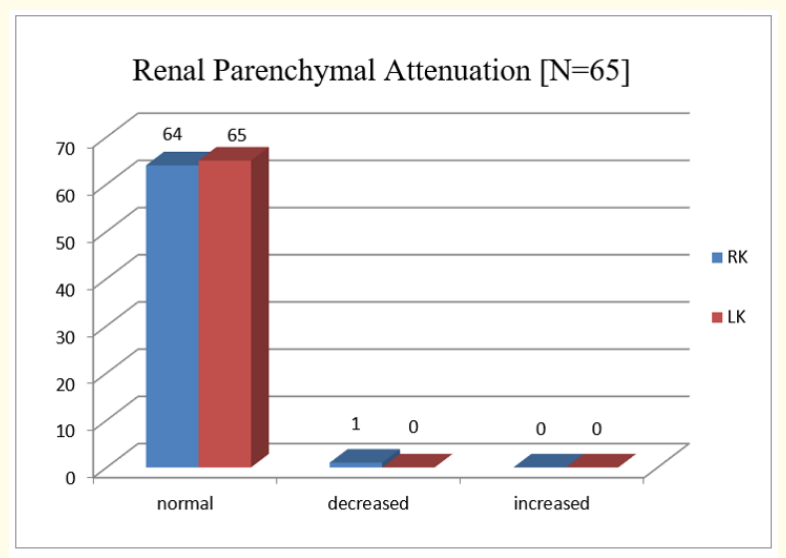

Figure $\mathbf{h}$

Incidental findings on ultrasound

\begin{tabular}{|l|c|}
\hline \multicolumn{1}{|c|}{ Findings } & Number of cases \\
\hline Hydronephrosis & 3 \\
\hline Simple cyst & 8 \\
\hline Complex cyst & 1 \\
\hline Non obstructive calculus & 5 \\
\hline Obstructive calculus & 1 (unilateral ureteric ) \\
\hline Pleural effusion & 2 \\
\hline Ascites & 2 \\
\hline
\end{tabular}

Table 8

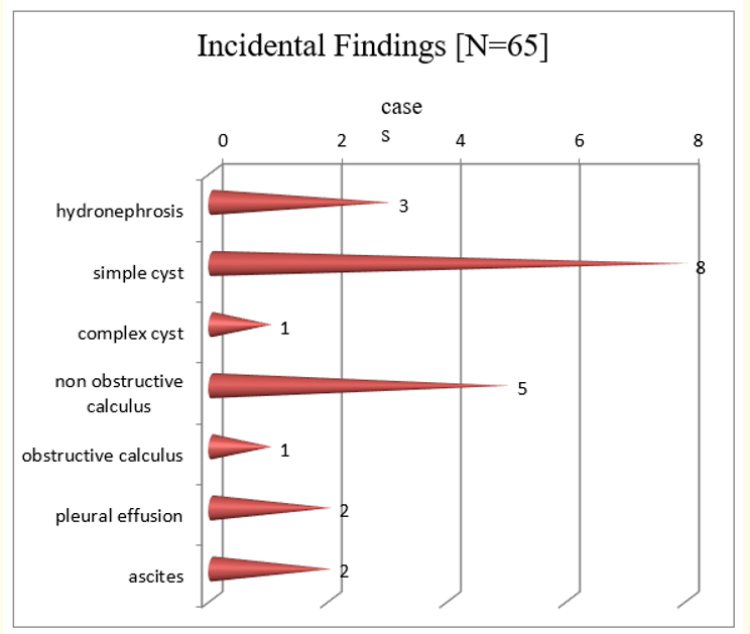

Figure $\mathbf{i}$

\begin{tabular}{|l|c|}
\hline \multicolumn{1}{|c|}{ Renal length } & Serum creatinine \\
\hline RRL pearson correlation (r) & .077 \\
2-tailed (p) & .542 \\
N & 65 \\
\hline LRL pearson correlation ( $\mathrm{r}$ ) & .131 \\
2-tailed (p) & .299 \\
N & 65 \\
\hline ARL pearson correlation (r) & .115 \\
2-tailed (p) & .363 \\
N & 65 \\
\hline
\end{tabular}

Table 9: Correlation between renal length and serum creatinine.

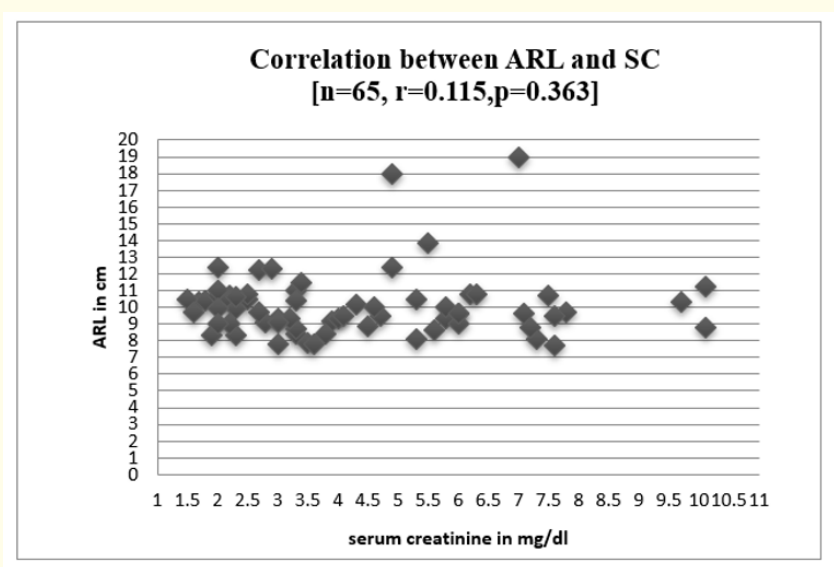

Figure j

There is no significant correlation between the average renal length (ARL) and serum creatinine by Pearson's and two tailed tests.

\begin{tabular}{|l|c|}
\hline \multicolumn{1}{|c|}{ Renal cortical echogenicity } & Serum creatinine \\
\hline Pearson Correlation $(\mathrm{r})$ & .865 \\
2-tailed $(\mathrm{p})$ & $.000^{*}$ \\
N & 65 \\
\hline
\end{tabular}

Table 10: Correlation between renal cortical echogenicity and serum creatinine.

*.correlation is significant at the 0.01 level (2-tailed). 


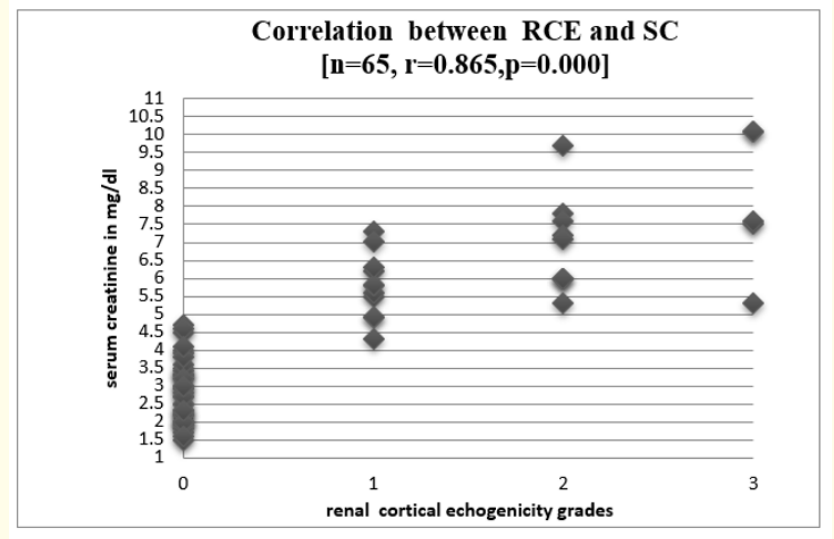

Figure k

There is significant correlation between renal cortical echogenicity and serum creatinine by two-tailed test at $\mathrm{p}=0.01$ level.

\begin{tabular}{|c|c|c|c|c|c|c|}
\hline \multirow{2}{*}{ RCE } & \multicolumn{5}{|c|}{ Creatinine Level (mg/d) } & \multirow{2}{*}{ N } \\
\cline { 2 - 6 } & Min & Max & Range & Var & Std. dev & \\
\hline Grade0 & 1.5 & 4.7 & 3.2 & .748 & .864 & 40 \\
Grade1 & 4.3 & 7.3 & 3 & .810 & .899 & 11 \\
Grade2 & 5.3 & 9.7 & 4.4 & 1.778 & 1.333 & 9 \\
Grade3 & 5.3 & 10.1 & 4.8 & 4.112 & 2.027 & 5 \\
\hline
\end{tabular}

Table 11: Relation between serum creatinine and RCE grades.

\begin{tabular}{|c|c|c|c|c|}
\hline Age group & $\mathbf{N}$ & Mean & $\begin{array}{c}\text { STD. } \\
\text { deviation }\end{array}$ & t-value \\
\hline $\begin{array}{l}\text { RRL Less than } 40 \\
\text { years } \\
\text { More than } 40 \text { years }\end{array}$ & $\begin{array}{l}14 \\
51\end{array}$ & $\begin{array}{l}10.5 \\
9.6\end{array}$ & $\begin{array}{l}1.21 \\
1.1\end{array}$ & 2.632 \\
\hline $\begin{array}{l}\text { LRL Less than } 40 \\
\text { years } \\
\text { More than } 40 \text { years }\end{array}$ & $\begin{array}{l}14 \\
51\end{array}$ & $\begin{array}{l}10.47 \\
9.65\end{array}$ & $\begin{array}{l}1.29 \\
1.29\end{array}$ & 2.111 \\
\hline $\begin{array}{l}\text { ARL Less than } 40 \\
\text { years } \\
\text { More than } 40 \text { years }\end{array}$ & $\begin{array}{l}14 \\
51\end{array}$ & $\begin{array}{c}10.48 \\
9.6\end{array}$ & $\begin{array}{l}1.21 \\
1.07\end{array}$ & 2.583 \\
\hline
\end{tabular}

Table 12: Correlation between renal length and age group.
There is a significant correlation between average renal length (ARL) and age by t-test at $t>2.58$. There is a slight decrease in the renal length with increase in age beyond the fourth decade.

\section{Discussion}

Our study is a prospective hospital based study; the population included 65 adult patients who presented to our nephrology department with raised serum creatinine levels.

\section{Demographics}

The age structure of the study population in this series varies from the $2^{\text {nd }}$ to $8^{\text {th }}$ decade. The youngest patient was 19 years old and the oldest was 89 years old, with a mean age of 54.6 years.

In our series of 65 cases, 45 (69.2\%) were males and 20 (30.8\%) were females (Table 1). Our study shows greater incidence of ARF among males than females which is similar to findings by Feest TG., et al. [1], who in a study has confirmed the predominance of acute renal failure in males compared to females.

Fifty one $(78.5 \%)$ patients in this study were above the age of 40 years (Table 3 ). The increased prevalence of acute renal failure in old age is said to be due to ageing, which is associated with a decline in renal function, approximately $50 \%$ of that of a 20 years old person [2]. It is also described that peak incidence of chronic diseases like diabetes, hypertension and glomerulonephritis are more common in age 65 to 74 years [3] and these are the risk factors in the development of acute renal failure in old age [4].

The maximum incidence of ARF in this series was found to be between 50-59 years. 15 patients (23.1\%) presented with ARF in the $5^{\text {th }}$ decade. Among males, 11 (25\%) presented between 70-79 years whereas in females, 7 (33.33\%) presented between 50-59 years (Table 4).

The effect of ageing on the kidney is well documented. Ageing is associated with a progressive loss of renal mass. Renal weight declines $20-30 \%$ between the age of 30 and 90 yrs [5,6].

\section{Renal size change and serum creatinine}

In our study, abdominal ultrasound was done in all the patients, 47 (72.3\%) patients had normal size kidneys, 15 (23.07\%) had small size kidneys, and 3 (4.61\%) had large sized kidneys (Table 5).

In our study there was no significant correlation between renal length and serum creatinine levels (Table 9). 
However there was a significant correlation between renal length and age group of the patient, wherein there was a slight decrease in the renal lengths in the forty and above age group patients (Table 12).

\section{Renal parenchyma echogenicity and serum creatinine}

The evaluation of the texture of the renal parenchyma and its brightness by ultrasound study in acute renal failure offers new and potentially useful information in formulating a diagnosis.

Several diseases associated with interstitial alterations have been reported [7] to cause an increase in cortical echogenicity with preservation of corticomedullary definition.

Focal interstitial changes tend to produce a minimal increase in cortical echogenicity while diffuse scarring produces greater increase in echogenicity, and the highest level echoes are seen with active interstitial infiltration.

In this series, when the cortical echogenicity was correlated with laboratory data, an overall significant accord was found between cortical echogenicity and the serum creatinine levels (Table 10). This is in contrast to the report by Moccia., et al. [8]. There was, however a wide range of variance present in each group and a normal cortical echogenicity was seen in patients with serum creatinine levels ranging from 1.5 to $5 \mathrm{mg} / \mathrm{dl}$ (Table 11).

\section{Renal parenchymal attenuation}

In our study, sixty four patients had normal parenchymal attenuation value in both kidneys, except for one who had a obstructive ureterolithiasis on the right side (Table 7). Renal attenuation on the side with lithiasis was lower compared to the opposite kidney.

Georgiades., et al. [9] emphasized the subjective evaluation of the attenuation difference, which is the visual identification of a low-attenuating kidney parenchyma in unilateral ureteric obstruction.

Goldman., et al. [10] in their study concluded that the attenuation difference between kidneys greater than or equal to $5.0 \mathrm{H}$ was a valuable sign and had diagnostic performance similar to other secondary signs of obstructive ureterolithiasis.

However in our study there was no significant correlation between renal parenchymal attenuation and serum creatinine levels.

\section{Limitations of Our Study}

Other known risk factors associated with ARF were not excluded and were not analyzed, which is the limitation of this study.

\section{Conclusion}

Our prospective study with 65 adult patients revealed that acute renal failure had a positive association with male gender and there was a gradual decrease in renal length with advancing age.

The renal cortical echogenicity on ultrasound had a positive relation with serum creatinine levels and reflected the severity of the disease process.

Non-enhanced computer tomography is a more sensitive modality in detecting other pathologies in the kidney, in particular renal and ureteric calculus. It also demonstrated a decrease in renal parenchymal attenuation in the obstructed kidney.

The limited responses of the kidney to various pathological insults may well remain a limitation to specific sonographic diagnosis, even when accurate quantitative measurements by ultrasound become available.

Sonographic characterization of tissue is at present an area of active research. Measurements of the degree of abnormality and the definition of unique tissue signatures are the goals.

\section{Bibliography}

1. Feest TG., et al. "Incidence of severe acute renal failure in adults: results of a community based study". BMJ 306 (1993): 481-483.

2. Steinman TI. "Kidney protection how to prevent or delay CRF". Giardiatrics 51 (1996): 28-35.

3. Lafayette RA. "Preventing disease progression in CRF". Lafayette 52 (1995): 178391.

4. Thadhani R., et al. "Acute renal failure". The New England Journal of Medicine 334 (1996): 1448-1460.

5. Tauchi H., et al. "Age changes in the human kidney of the different races". Gerontologia 17 (1971): 87-97.

6. Brown WW., et al. "Aging and the kidney". Archives of Internal Medicine 146 (1986): 1790-6.

7. Hricak H., et al. "Renal parenchymal disease: Sonographic-histologic correlation". Radiology 144 (1982): 141-147.

8. Moccia WA., et al. "Evaluation of chronic renal failure by digital gray-scale ultrasound". Urology and Radiology 2 (1980): 1-17. 
9. Georgiades CS., et al. "Differences of renal parenchymal attenuation for acutely obstructed and unobstructed kidneys on unenhanced helical CT: a useful secondary sign?" American Journal of Roentgenology 176 (2001): 965-968.

10. Suzan M Goldman., et al. "Diagnostic Value of Attenuation Measurements of the Kidney on Unenhanced Helical CT of Obstructive Ureterolithiasis". American Journal of Roentgenology 182 (2004): 1251-1254.

\section{Volume 5 Issue 7 July 2021}

(C) All rights are reserved by Dr. Moscow Mani S A MBBS

MD. 